
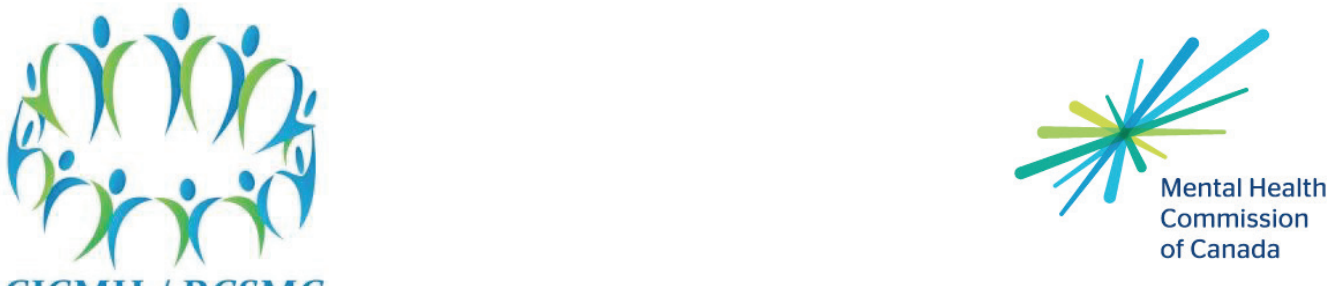

Commission de la santé mentale

CJCMH / RCSMC

du Canada

\title{
Responses to the Mental Health Strategy for Canada: Canadian and International Perspectives on Mobilizing Change
}

Issue II

\section{Call for Papers}

for a special issue of the

\section{Canadian Journal of Community Mental Health \\ www.cjcmh.com}

Due September $1^{\text {st }} 2016$

[La version française suit.]

With the release of the historic document "Changing Directions, Changing Lives: The Mental Health Strategy for Canada", the Mental Health Commission of Canada (MHCC) has presented a blue print for change in how we promote mental health, prevent mental illness and support those whose lives are affected by mental illness.

Following the release of the first special issue, the Journal is now accepting submissions for the second of two special issues, which is targeted for release in Spring 2017. The first of the two special issues emphasized the foundations for the strategy and the conditions necessary to create lasting and meaningful change. The second special issue will focus on implementing transformative change. 


\section{Issue 2: Making Change Happen}

Submissions may include descriptions of exemplary programmes, policy analyses, theoretical articles, research reports, literature reviews, 'First Voice' reflections on experiences and lessons learned, advice on how to scale up promising practices and how to address policy and funding gaps. Example topics are provided in the chart below.

\section{Types of Articles}

The issues will feature two sections: Searchlight Articles, and Spotlight Articles. Searchlight articles will consist of longer articles (4000-6000 words) that take a broad lens and may provide conceptual analysis, review of the literature and/or discussion of an issue related to the successful implementation of the strategy. The second section will include Spotlight Articles, which are shorter, more focused contributions (18004000 words) that provide particular examples drawing from the world of policy, personal experience and clinical practice. These may be examples of implementation that can be adopted or adapted elsewhere in the country and they may include narrative accounts. Please follow the instructions to authors on our website: http://cjcmh.com/page/cjcmh/itas

\section{Who can Submit Articles?}

Contributions are invited from Canadian and international scholars, practitioners, policy makers and people with lived experience of mental illness, including family caregivers. We hope to engage provinces and territories in reporting work underway in their jurisdictions, provide tools to encourage uptake of the strategy, mobilize individual communities and the broader mental health community in Canada.

\section{Potential Topics}

Potential topics for the second issue and a tentative schedule is provided in the table below. 


\begin{tabular}{|l|}
\hline Potential Topics \\
\hline Moving from Strategy to Action Plans \\
\hline Lessons from Other Jurisdictions \\
\hline Relationship between the Strategy and Provincial/Territorial plans \\
\hline Implementing Recovery \\
\hline Scalability of Innovation \\
\hline Social Determinants, health equity \\
\hline Involving people with lived experience \\
\hline Use of Guidelines \\
\hline Using data to guide implementation \\
\hline Measuring Community Capacity and Needs \\
\hline Mental Health and Housing \\
\hline Bridging Systems: Across Services, Sectors and the Lifespan \\
\hline Primary Health Care \\
\hline Stigma Reduction \\
\hline Mobilizing Leadership, Fostering Collaboration in the Canadian Political Context \\
\hline Funding \\
\hline Current State of Implementation Barriers/Opportunities \\
\hline Social Inclusion and Supported Employment \\
\hline School-based Mental Health \\
\hline Developing the Mental Health Workforce \\
\hline Child and Youth Mental Health \\
\hline The Role of Peer Support in Recovery \\
\hline What's next for the MHCC-2017 and beyond \\
\hline
\end{tabular}

As well we invite readers to provide commentary/letters to the editors on articles that were published in the first special issue.

Information about the journal can be found on the website: http://cjcmh.com/journal/cjcmh

Individuals intending to submit papers are encouraged to contact the editors listed below to assess the appropriateness of proposed submissions and answer questions. The deadline for complete submissions for the second issue is September 1, 2016. Early submissions and inquiries will be appreciated. 


\title{
Submissions in English:
}

Guest Editors: Steve Lurie, CMHA Toronto, Gillian Mulvale, McMaster University slurie@cmha-toronto.netrmulvale@mcmaster.ca

\author{
Submissions in French \\ Tania Lecomte, Université de Montréal \\ tania.lecomte@umontreal.ca
}



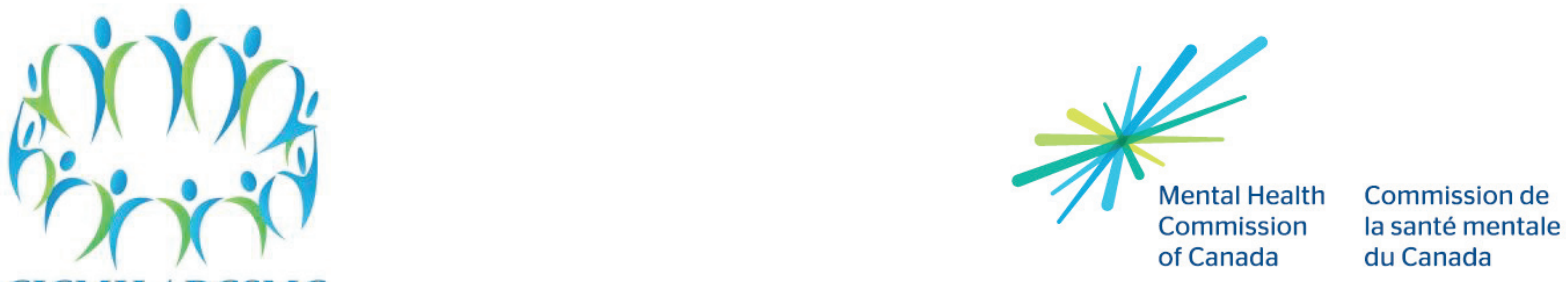

CJCMH / RCSMC

\author{
Mettre en œuvre la stratégie \\ en matière de santé mentale pour le Canada : \\ des perspectives canadiennes et d'ailleurs dans le monde
}

$2^{\mathrm{e}}$ numéro

\begin{abstract}
Appel d'articles
pour un numéro spécial de

Canadian Journal of Community Mental Health/

Revue canadienne de santé mentale communautaire

www.cjcmh.com
\end{abstract}

\title{
Date limite : le 1 septembre 2016
}

Avec la publication de Changer les orientations, changer des vies - Stratégie en matière de santé mentale pour le Canada, un document marquant, la Commission de la santé mentale du

Canada a présenté un plan visant à transformer nos façons de promouvoir et de prévenir la santé mentale et de soutenir les personnes vivant avec un trouble mental ou une maladie mentale.

À la suite de la publication du premier numéro spécial traitant de ce sujet, la revue souhaite recevoir des articles pour le deuxième numéro, dont la publication est prévue au printemps de 2017. Le premier numéro a mis l'accent sur les fondements de la stratégie et sur les conditions 
nécessaires à la réalisation des changements profonds et durables à mettre en place. Le deuxième numéro portera sur la réalisation de ces changements.

Thème : «Réaliser les changements »

Nous souhaitons recevoir des articles offrant des exemples de mesures et de programmes d'application de la stratégie, des analyses de politiques, des textes théoriques, des rapports de recherche, des revues de littérature, des réflexions sur des expériences personnelles offrant des enseignements utiles, des conseils sur des pratiques prometteuses à reproduire et des propositions de solutions aux lacunes du système actuel sur le plan des politiques et du financement. Des exemples de sujets sont présentés plus bas.

Types d'articles

Le numéro comportera deux sections : "Sous les réflecteurs " et " Sous les projecteurs". Les articles de la section "Sous les réflecteurs " (de 4000 à 6000 mots) seront des textes de fond comme des analyses conceptuelles, des revues de littérature ou la présentation détaillée d'applications réussies de la stratégie. Les articles de la section "Sous les projecteurs " (de 1800 à 4000 mots) traiteront plutôt d'exemples liés au domaine des politiques publiques, d'expériences personnelles ou de pratiques cliniques qui pourraient être adaptés ailleurs ou dans d'autres contextes, et qui peuvent prendre la forme d'un simple compte rendu.

Veuillez suivre les normes de présentation des articles, que vous trouverez dans la section « Recommandation aux auteurs » de notre site Web (http://cjcmh.com/page/cjcmh/itas).

Qui peut soumettre un article

Nous faisons appel à des chercheurs canadiens ou d'ailleurs dans le monde, à des praticiens, à des décideurs politiques ainsi qu'à des personnes vivant ou ayant vécu avec une maladie mentale et à des aidants naturels qui soutiennent des personnes ayant un problème de santé mentale. Nous souhaitons également que les administrations provinciales et les territoriales soumettent des textes sur ce qu'elles font actuellement et proposent ainsi des outils qui pourraient permettre de mieux appliquer la stratégie et de mobiliser les communautés canadiennes et l'ensemble du milieu de la santé mentale au Canada. 


\section{Exemples de sujets}

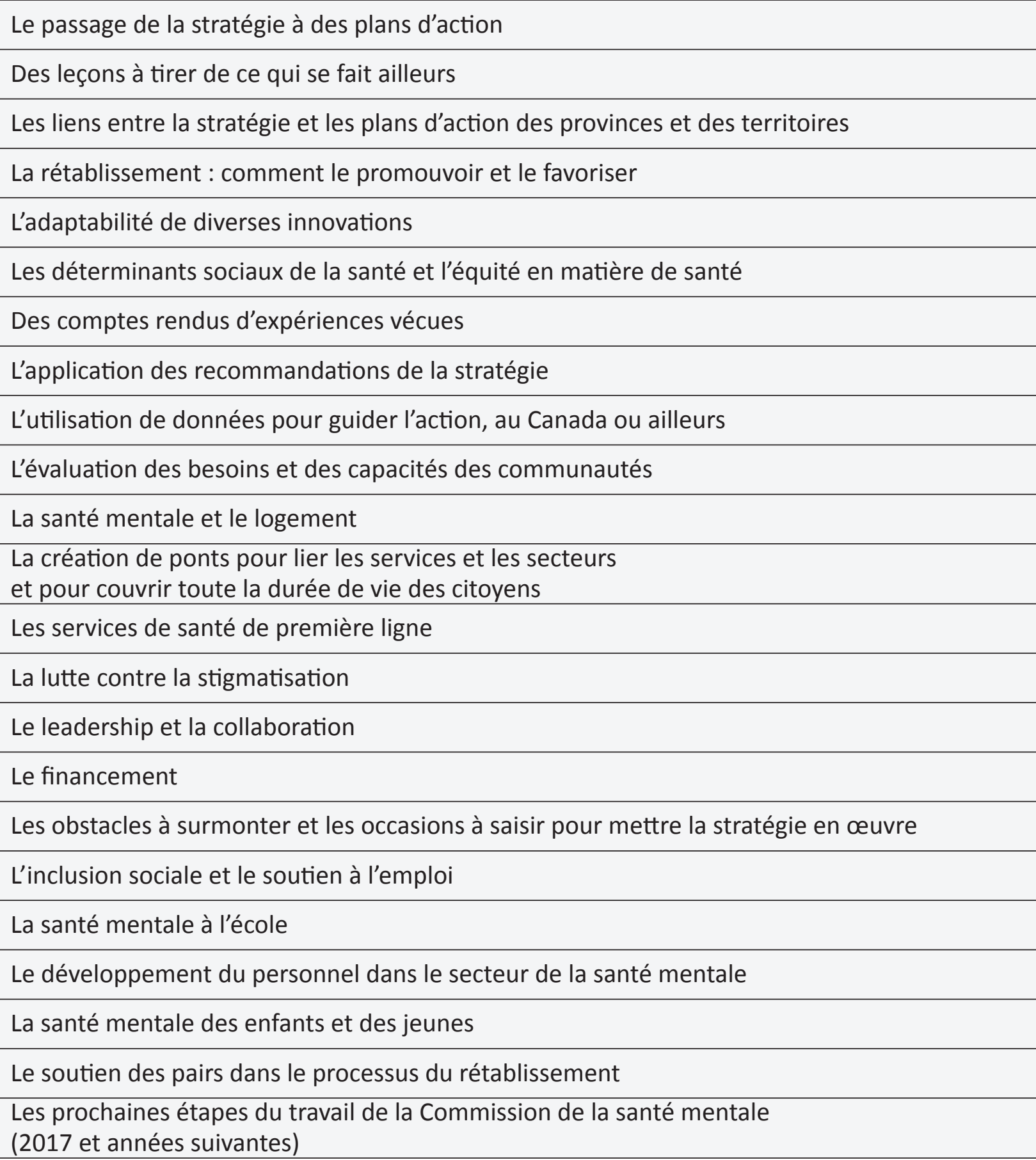

Nous invitons également nos lecteurs à nous envoyer des lettres ou des commentaires sur les articles du premier numéro spécial. 
Pour plus d'information sur la revue, consultez notre site Web (http://cjcmh.com/journal/cjcmh).

Avant de soumettre un article, nous vous invitons à communiquer avec l'une des personnes responsables ci-dessous, qui pourra vérifier la pertinence de votre texte et répondre à vos questions. La date limite pour soumettre un article est le $1^{\mathrm{er}}$ septembre0 2016. Nous apprécierons bien sûr que vous le fassiez avant cette date.

\section{Articles en anglais :}

Directeurs invités Steve Lurie, ACSM Toronto, slurie@cmha-toronto.net

Gillian Mulvale, Université McMaster, mulvale@mcmaster.ca

Articles en français : Tania Lecomte, Université de Montréal, tania.lecomte@umontreal.ca 